\section{A "Declaração de Nascido Vivo" como orientadora de ações de saúde em nível local}

\section{The "Born Alive Report" as a health guide at local level}

Emília de Faria Carniel 1 Maria Ângela Reis de Góes Monteiro Antonio 2 Mirna Ruth M. Lima e Mota 3

André Moreno Morcillo 4

Maria de Lurdes Zanolli 5

1,2,4,5 Departamento de Pediatria. Faculdade de Ciências Médicas. Universidade Estadual de Campinas. Cidade Universitária Zeferino Vaz. Rua Alexander Fleming, 181. Caixa Postal 6.111. Campinas, SP, Brasil. CEP: 13.084-971

3 Centro de Saúde São Quirino, Campinas, SP, Brasil

\begin{abstract}
Objectives: to describe the profile of mothers and newborns in the vicinities of a Health Clinic based on the "Born Alive Report" (BAR).

Methods: cross-sectional descriptive study analyzing 400 BAR of a Health Clinic in the city of Campinas, in 1999. Mothers' profiles were described according to: age, education, job, marital status, number of children, frequency of medical visits during prenatal care, type of delivery; the newborns were described according to: birth weight and pregnancy age. The assessment of the association between variables was performed through the chi square test and crude odds ratios (OR) were calculated. For the variables of mothers age and type of delivery a model of logistical regression was used to obtain adjusted OR values.

Results: mothers: 17,8\% adolescents, $54,9 \%$ had not finished elementary school, 26\% had no husband or companion, 40,2\% no income, 44,3\% with up to six medical visits during prenatal care. Children: $6,5 \%$ premature, 50,3\% C-sections, 9\% weighing up to 2.499 grams. Risks were inferred related to adolescents without husbands, without income and without information related to jobs. It was noted that women without income and low educational level were protected from $C$-sections.

Conclusions: "Born Alive Reports" furnished input on mothers and newborns and demonstrated to be an adequate tool to monitor local reality, enabling the planning of actions.
\end{abstract}

Key words Newborns, Information systems, Pregnant women

\section{Resumo}

Objetivos: descrever o perfil de mães e recémnascidos da área de um Centro de Saúde (CS), partindo das "Declarações de Nascidos Vivos" (DNVS).

Métodos: estudo descritivo transversal que analisou 400 DNV de um CS de Campinas, em 1999. Identificou-se o perfil das mães por: idade, escolaridade, ocupação, situação conjugal, número de filhos, freqüencia ao pré-natal; tipo de parto; e dos recémnascidos por: peso de nascimento e idade gestacional. Na avaliação da associação entre variáveis empregou-se o teste do qui-quadrado e calcularam-se os valores de odds ratio (OR) brutos. Para as variáveis idade da mãe e tipo de parto empregou-se o modelo de regressão logística a fim de obter valores de OR ajustados.

Resultados: mães: 17,8\% adolescentes, 54,9\% com primeiro grau incompleto, $26 \%$ sem companheiro, 40,2\% sem renda, 44,3\% com até seis consultas de pré-natal. Crianças: $6,5 \%$ prematuras, $50,3 \%$ de cesárea, 9\% pesando até 2.499 gramas. Evidenciaram-se riscos para as adolescentes sem companheiro, sem renda e sem informação sobre a ocupação. Observou-se que mulheres sem renda e com baixa escolaridade foram protegidas dos partos cesáreos.

Conclusões: as DNVS possibilitaram conhecer mães e RN e se revelaram um instrumento adequado para monitoramento da realidade local, possibilitando planejamento de ações.

Palavras-chave Recém-nascidos, Sistemas de Informações, Gestantes 


\section{Introdução}

O Sistema de Informações sobre Nascidos Vivos (SINASC) do Ministério da Saúde (MS) foi implantado no Brasil a partir de 1990, com o intuito de obterem-se informações fidedignas sobre gestação, nascimentos e características maternas, podendo-se assim, através da elaboração de diversos indicadores, planejar ações de saúde destinadas à população materno-infantil. ${ }^{1}$ Desde então, a "Declaração de Nascidos Vivos" (DNV) tornou-se o instrumento obrigatório para a coleta de dados. ${ }^{2}$

Em Campinas, a implantação do SINASC ocorreu em 1991, sob a coordenação da Regional Estadual de Saúde (atual DIR XII), acatando as diretrizes do MS, podendo, com isso, viabilizar políticas e ações para mães e recém-nascidos (RN). A partir de 1997 a Secretaria Municipal de Saúde assumiu esta tarefa e passou a encaminhar as DNVs aos Centros de Saúde (CS) visando principalmente a vigilância dos $\mathrm{RN}$ de risco.

Em um CS do município de Campinas, optou-se por ampliar a vigilância para todos os RN e puérperas de sua área de abrangência, passando-se a realizar visitas domiciliares (VD) para este grupo, com o intuito de identificar as necessidades das crianças e mães através de possíveis queixas e dúvidas, observar a família, o ambiente e as condições de vida. Além disso, fazer orientações no domicílio e encaminhar ao CS para seguimento da mãe e da criança, conforme recomendação dos programas da criança e da mulher. 3,4

As VDs, supervisionadas por docentes (pediatra e enfermeira), são realizadas por alunos do quarto ano de graduação em medicina e residentes do primeiro ano dentro do estágio de pediatria social da Faculdade de Ciências Médicas da Universidade Estadual de Campinas (UNICAMP).5,6

Para a continuidade desta atividade e implementação de novas ações com RN e mães, houve necessidade de um conhecimento mais abrangente desta população.

O objetivo deste trabalho é descrever o perfil das mães e RN da área de abrangência deste CS.

\section{Métodos}

Realizou-se um estudo descritivo transversal, que analisou todas as DNVs enviadas pela Secretaria de Saúde ao CS São Quirino, localizado em Campinas, SP, Brasil, em 1999, cujas mães eram residentes na área de abrangência dessa unidade, totalizando 400 nascidos vivos.
Esse CS é um dos 46 da rede municipal, localizado na região leste de Campinas e responsável por uma população de cerca de 30.000 habitantes, que engloba desde os grupos de classe média alta, residentes em condomínios fechados, de luxo, até aqueles residentes em locais sem infra-estrutura urbana, sendo que nestas favelas residem um terço da população da área. Estima-se que aproximadamente $70 \%$ da população da região seja usuária do Sistema Único de Saúde (SUS).

As DNVs são preenchidas nos hospitais que realizam partos no município e pelo cartório onde a criança for registrada, caso o parto ocorra fora do serviço de saúde. Os profissionais são treinados conforme manual do MS e periodicamente são realizadas reuniões com as instituições para atualização de orientações no preenchimento dos formulários.

A Secretaria recolhe as DNVs, verifica o preenchimento dos dados e, estando correto este preenchimento, encaminha ao CS de referência da mãe e da criança. Caso falte anotação de algum campo, é solicitada à instituição completar o preenchimento.

O perfil das mães e dos recém-nascidos foi identificado através de características sócio-demográficas (faixa etária, escolaridade, ocupação, situação conjugal das mães), gestacionais (número de filhos e freqüência ao pré-natal) e relacionadas ao parto e às condições do nascimento (peso do recém-nascido, tipo de parto e idade gestacional). Foram consideradas variáveis dependentes a idade da mãe $(<20$ anos e $>20$ anos $)$, o peso de nascimento $(<2.500,2.500 \mid-$ 3.000 e $>3.000$ gramas) e o tipo de parto (vaginal, cesárea).

Foram consideradas variáveis independentes: ocupação (com renda, sem informação sobre atividade remunerada da mãe e sem renda), situação conjugal (com companheiro, sem companheiro e sem informação), escolaridade da mãe (até primeiro grau incompleto, primeiro grau completo, $>$ segundo grau), duração da gestação em semanas (28 a 36 e 37 a 41), número de consultas de pré-natal ( $<7$ e $\geq 7$ ) e número de filhos $(1,2$ e $\geq 3)$. Na análise estatística os grupos de escolaridade - até primeiro grau incompleto e primeiro grau completo - foram reunidos devido à sua baixa frequiência. Foram excluídos os casos em que havia falta de informação para alguma das variáveis.

Estudou-se a associação entre as variáveis dependentes e independentes utilizando-se o teste quiquadrado e os valores de odds ratio bruto. Para análise das variáveis dicotômicas (tipo de parto e idade da mãe) empregou-se o modelo de regressão logística (método backward stepwise) usando-se o 
software SPSS 10.0, com o objetivo de se obter valores de odds ratio ajustados.

Este trabalho foi aprovado pelo Comitê de Ética da Faculdade de Ciências Médicas da UNICAMP (Parecer 362/2002).

\section{Resultados}

Na Tabela 1, onde são apresentadas as características das mães em relação à idade, escolaridade, situação conjugal e ocupação observa-se que: $17,8 \%$ eram adolescentes ( $<20$ anos), 54,9\% não completaram o primeiro grau, $26 \%$ não tinham companheiro fixo, e $40,2 \%$ não exerciam atividade remunerada.

Tabela 1

Distribuição de freqüência das mães residentes na área de abrangência do Centro de Saúde São Quirino, em relação às características sócio-demográficas. Campinas SP, 1999.

\begin{tabular}{lrr}
\hline Características sócio-demográficas & N & $\%$ \\
\hline Idade da mãe & & 17,8 \\
$\quad<20$ anos & 324 & 82,2 \\
$\geq 20$ anos & 394 & 100,0 \\
Total* & & \\
& & 54,9 \\
Escolaridade da mãe & 208 & 9,5 \\
Primeiro grau incompleto & 36 & 35,6 \\
Primeiro grau completo & 135 & 100,0 \\
$\geq 2^{\circ}$ grau & 379 & \\
Total** & & 40,0 \\
& & 26,0 \\
Situação conjugal & 160 & 34,0 \\
Com companheiro & 104 & 100,0 \\
Sem companheiro & 136 & 100,0 \\
Sem informação & 400 & \\
Total & & 30,5 \\
Ocupação & & 40,2 \\
Com renda & 122 & 161 \\
Sem renda & 117 & \\
Sem informação & 400 & \\
\hline
\end{tabular}

* Excluídos seis por falta de informação; ** Excluídos 21 por falta de informação.

Fonte: Sistema de Informações sobre Nascidos Vivos. Centro de Saúde São Quirino: janeiro a dezembro, 1999. Campinas, SP.

Quanto às características dos partos e dos nascimentos (Tabela 2), verificou-se que 6,5\% das crianças nasceram prematuras (<37 semanas), 50,3\% de parto cesárea, $9 \%$ com peso inferior a 2.500 gramas e $24,3 \%$ com peso insuficiente $(2.500$ a 2.999 gramas). Ainda em relação às características gestacionais observou-se que $44,3 \%$ das mulheres fizeram menos do que sete consultas de pré-natal e $69,9 \%$ tinham até dois filhos. 
Distribuição de freqüência dos nascimentos ocorridos na área de abrangência do Centro de Saúde São Quirino em relação às características gestacionais, relacionadas ao parto e às condições de nascimento. Campinas, SP, 1999.

\begin{tabular}{|c|c|c|}
\hline $\begin{array}{l}\text { Características gestacionais e relacionadas ao parto e às } \\
\text { condições de nascimento }\end{array}$ & $\mathbf{N}$ & $\%$ \\
\hline \multicolumn{3}{|l|}{ Duração da gestação } \\
\hline $28-36$ & 26 & 6,5 \\
\hline $37-41$ & 373 & 93,5 \\
\hline Total* & 399 & 100,0 \\
\hline \multicolumn{3}{|l|}{ Número consultas no pré-natal } \\
\hline$<7$ & 172 & 44,3 \\
\hline$\geq 7$ & 216 & 55,7 \\
\hline Total** & 388 & 100,0 \\
\hline \multicolumn{3}{|l|}{ Tipo de parto } \\
\hline Vaginal & 199 & 49,7 \\
\hline Cesárea & 201 & 50,3 \\
\hline Total & 400 & 100,0 \\
\hline \multicolumn{3}{|l|}{ Peso de nascimento (gramas) } \\
\hline$<2.500$ & 36 & 9,0 \\
\hline 2.500 I- 2.999 & 97 & 24,3 \\
\hline$\geq 3.000$ & 267 & 66,7 \\
\hline Total & 400 & 100,0 \\
\hline \multicolumn{3}{|l|}{ Número de filhos } \\
\hline 1 & 144 & 37,3 \\
\hline 2 & 126 & 32,6 \\
\hline$\geq 3$ & 116 & 30,1 \\
\hline Total $* * *$ & 386 & 100,0 \\
\hline
\end{tabular}

* Excluído um por falta de informação; ** Excluídos 12 por falta de informação; *** Excluídos 14 por falta de informação.

Fonte: Sistema de Informações sobre Nascidos Vivos Centro de Saúde São Quirino: janeiro a dezembro, 1999. Campinas, SP.

Na Tabela 3 são apresentadas as distribuições de freqüências dos grupos de peso ao nascimento em relação à duração da gestação, número de consultas de pré-natal, tipo de parto, situação conjugal, ocupação, escolaridade e idade materna. Observa-se a associação entre peso de nascimento e duração da gestação $(\mathrm{p}<0,001)$. 
Distribuição de freqüência dos nascidos vivos da área de abrangência do Centro de Saúde São Quirino em relação ao peso de nascimento e duração da gestação, número de consultas no pré-natal, tipo de parto, situação conjugal, ocupação, escolaridade e idade da mãe. Campinas, SP, 1999.

\begin{tabular}{|c|c|c|c|c|c|c|c|c|}
\hline \multirow{2}{*}{ Categorias de análise } & \multirow[b]{2}{*}{$\mathbf{N}$} & \multicolumn{2}{|c|}{$\geq 3.000 \mathrm{~g}$} & \multicolumn{2}{|c|}{$2.500-2.999 \mathrm{~g}$} & \multicolumn{2}{|c|}{$<2.500 \mathrm{~g}$} & \multirow{2}{*}{$\mathbf{p}$} \\
\hline & & $\mathrm{n}$ & $\%$ & $\mathrm{n}$ & $\%$ & $\mathrm{n}$ & $\%$ & \\
\hline \multicolumn{9}{|l|}{ Duração } \\
\hline $37-41$ & 373 & 264 & 70,8 & 90 & 24,1 & 19 & 5,1 & \\
\hline $28-36$ & 26 & 3 & 11,5 & 7 & 26,9 & 16 & 61,6 & $p<0,001$ \\
\hline \multicolumn{9}{|l|}{ Número de consultas } \\
\hline$\geq 7$ & 216 & 148 & 68,5 & 54 & 25,0 & 14 & 6,5 & \\
\hline$<7$ & 172 & 113 & 65,7 & 40 & 23,3 & 19 & 11,0 & $p=0,275$ \\
\hline \multicolumn{9}{|l|}{ Tipo de parto } \\
\hline Vaginal & 199 & 131 & 65,8 & 47 & 23,6 & 21 & 10,6 & \\
\hline Cesárea & 201 & 136 & 67,6 & 50 & 24,9 & 15 & 7,5 & $p=0,555$ \\
\hline \multicolumn{9}{|l|}{ Situação conjugal } \\
\hline Com companheiro & 160 & 112 & 70,0 & 38 & 23,7 & 10 & 6,3 & \\
\hline Sem companheiro & 104 & 73 & 70,2 & 25 & 24,0 & 6 & 5,8 & $p=0,066$ \\
\hline Sem informação & 136 & 82 & 60,3 & 34 & 25,0 & 20 & 14,7 & \\
\hline \multicolumn{9}{|l|}{ Ocupação } \\
\hline Com renda & 122 & 83 & 68,0 & 31 & 25,4 & 8 & 6,6 & \\
\hline Sem renda & 161 & 108 & 67,1 & 39 & 24,2 & 14 & 8,7 & $p=0,700$ \\
\hline Sem informação & 117 & 76 & 65,0 & 27 & 23,0 & 14 & 12,0 & \\
\hline \multicolumn{9}{|l|}{ Escolaridade } \\
\hline$>$ Segundo grau & 135 & 99 & 73,3 & 31 & 23,0 & 5 & 3,7 & \\
\hline Até primeiro grau completo & 244 & 160 & 65,6 & 58 & 23,8 & 26 & 10,6 & $p=0,051$ \\
\hline \multicolumn{9}{|l|}{ Idade da mãe } \\
\hline$\geq 20$ & 324 & 220 & 67,9 & 76 & 23,5 & 28 & 8,6 & \\
\hline$<20$ & 70 & 42 & 60,0 & 21 & 30,0 & 7 & 10,0 & $p=0,435$ \\
\hline
\end{tabular}

p = significância (teste qui-quadrado)

Fonte: Sistema de Informações sobre Nascidos Vivos. Centro de Saúde São Quirino: janeiro a dezembro, 1999. Campinas, SP.

Na avaliação da idade materna em relação à situação conjugal, escolaridade da mãe, número de consultas pré-natal, tipo de parto, duração da gestação, ocupação e peso de nascimento encontraramse associações entre idade e: situação conjugal ( $\mathrm{p}=$ $0,004)$, escolaridade materna $(\mathrm{p}=0,041)$, tipo de parto $(p=0,01)$ e ocupação ( $<<0,001)$ (Tabela 4). Foram selecionadas para regressão logística as variáveis situação conjugal, ocupação e escolaridade materna, permanecendo no modelo a ocupação e a situação conjugal. Para a ocupação, o grupo sem renda teve ORaj = 4,98 [IC95\% 2,20 - 11,24] e o grupo sem informação da ocupação ORaj = 3,99 [IC95\% 1,11 14,36]). Para a situação conjugal, o grupo de adolescentes sem companheiro apresentou ORaj $=2,67$ [IC95\% 1,35 - 5,27]). 
Distribuição de freqüência e odds ratio da idade materna em relação às variáveis situação conjugal, escolaridade, número de consultas, tipo de parto, duração da gestação, ocupação e peso de nascimento. Centro de Saúde São Quirino, Campinas, SP, 1999.

\begin{tabular}{|c|c|c|c|c|c|c|c|c|c|}
\hline \multirow{2}{*}{ Categorias de análise } & & \multirow[b]{2}{*}{$\mathbf{N}$} & \multicolumn{2}{|c|}{$<20$ anos } & \multicolumn{2}{|c|}{$\geq 20$ anos } & \multirow{2}{*}{ OR } & \multirow{2}{*}{ IC95\% } & \multirow{2}{*}{ p } \\
\hline & & & $\mathrm{n}$ & $\%$ & $\mathrm{n}$ & $\%$ & & & \\
\hline \multirow[t]{4}{*}{ Situação conjugal } & Sem companheiro & 103 & 29 & 28,1 & 74 & 71,9 & 2,72 & $1,38-5,40$ & \\
\hline & & & & & & & $2,67 *$ & $1,35-5,27$ & \\
\hline & Com companheiro & 159 & 20 & 12,6 & 139 & 87,4 & 1,00 & & 0,004 \\
\hline & Sem informação & 132 & 21 & 15,9 & 111 & 84,1 & 1,31 & $0,65-2,68$ & \\
\hline \multirow[t]{2}{*}{ Escolaridade da mãe } & Até primeiro grau completo & 239 & 50 & 20,9 & 189 & 79,1 & 1,95 & $1,02-3,76$ & 0,041 \\
\hline & $\geq$ Segundo grau & 134 & 16 & 11,9 & 118 & 88,1 & 1,00 & & \\
\hline Número de consultas & $<7$ & 168 & 29 & 17,3 & 139 & 82,7 & 1,07 & $0,60-1,89$ & 0,920 \\
\hline pré-natal & $\geq 7$ & 214 & 35 & 16,4 & 179 & 83,6 & 1,00 & & \\
\hline \multirow[t]{2}{*}{ Tipo de parto } & Vaginal & 197 & 45 & 22,8 & 152 & 77,2 & 1,00 & & \\
\hline & Cesárea & 197 & 25 & 12,7 & 172 & 87,3 & 0,49 & $0,28-0,87$ & 0,010 \\
\hline \multirow[t]{2}{*}{ Duração da gestação } & $\geq 37$ semanas & 369 & 63 & 17,1 & 306 & 82,9 & 1,00 & & \\
\hline & $<37$ semanas & 25 & 7 & 28,0 & 18 & 72,0 & 1,89 & $0,68-5,05$ & $0,130^{* *}$ \\
\hline \multirow[t]{5}{*}{ Ocupação } & Com renda & 122 & 8 & 6,6 & 114 & 93,4 & 1,00 & & \\
\hline & Sem renda & 158 & 44 & 27,8 & 114 & 72,2 & 5,50 & $2,36-13,28$ & $<0,001$ \\
\hline & & & & & & & $4,98^{*}$ & $2,20-11,24$ & \\
\hline & Sem informação & 114 & 18 & 15,8 & 96 & 84,2 & 2,67 & $1,04-7,04$ & \\
\hline & & & & & & & $3,99 *$ & $1,11-14,36$ & \\
\hline \multirow[t]{3}{*}{ Peso de nascimento } & $<2.500 \mathrm{~g}$ & 35 & 7 & 20,0 & 28 & 80,0 & 1,31 & $0,49-3,41$ & \\
\hline & $2.500 \mathrm{l}-3.000 \mathrm{~g}$ & 97 & 21 & 21,6 & 76 & 78,4 & 1,45 & $0,77-2,70$ & 0,430 \\
\hline & $\geq 3.000 \mathrm{~g}$ & 262 & 42 & 16,0 & 220 & 84,0 & 1,00 & & \\
\hline
\end{tabular}

OR = Odds ratio; IC95\% = Intervalo de confiança de 95\% do odds ratio; $\mathrm{p}=$ significância (teste qui-quadrado), * Odds ratio ajustado;

** Teste exato de Fisher.

Fonte: Sistema de Informações sobre Nascidos Vivos. Centro de Saúde São Quirino: janeiro a dezembro, 1999. Campinas, SP.

Considerando-se o tipo de parto, foram selecionadas para regressão logística as variáveis, situação conjugal $(\mathrm{p}=0,17)$, escolaridade da mãe $(\mathrm{p}=$ $0,002)$, ocupação $(\mathrm{p}<0,0004)$ e idade da mãe $(\mathrm{p}=$ $0,012)$, permanecendo no modelo somente a ocu- pação e a escolaridade, onde se destacaram os grupos sem renda: ORaj = 0,41 [IC95\% 0,24 - 0,69] e até primeiro grau completo: ORaj $=0,57$ [IC95\% $0,36-0,90]$. Os valores dos odds ratio brutos são apresentados na Tabela 5. 
Distribuição de freqüência e valores de odds ratio brutos do tipo de parto em relação à situação conjugal, escolaridade, ocupação e idade da mãe. Centro de Saúde São Quirino, Campinas, SP, 1999.

\begin{tabular}{|c|c|c|c|c|c|c|c|c|}
\hline \multirow{2}{*}{ Categorias de análise } & \multirow[b]{2}{*}{$\mathbf{N}$} & \multicolumn{2}{|c|}{ Cesárea } & \multicolumn{2}{|c|}{ Vaginal } & \multirow{2}{*}{ OR } & \multirow{2}{*}{ IC95\% } & \multirow{2}{*}{$\mathbf{p}$} \\
\hline & & $\mathrm{n}$ & $\%$ & $\mathrm{n}$ & $\%$ & & & \\
\hline \multicolumn{9}{|l|}{ Situação conjugal } \\
\hline Sem companheiro & 104 & 45 & 43,3 & 59 & 56,7 & 0,62 & $0,37-1,06$ & 0,170 \\
\hline Falta de informação & 136 & 68 & 50,0 & 68 & 50,0 & 0,82 & $0,50-1,33$ & \\
\hline Com companheiro & 160 & 88 & 55,0 & 72 & 45,0 & 1,00 & & \\
\hline \multicolumn{9}{|l|}{ Escolaridade da mãe } \\
\hline \multirow[t]{2}{*}{ Até primeiro grau completo } & 244 & 109 & 44,7 & 135 & 55,3 & 0,51 & $0,32-0,79$ & 0,002 \\
\hline & & & & & & 0,57 * & $0,36-0,90$ & \\
\hline$>$ Segundo grau & 135 & 83 & 61,5 & 52 & 38,5 & 1,00 & & \\
\hline \multicolumn{9}{|l|}{ Ocupação } \\
\hline \multirow[t]{2}{*}{ Sem renda } & 161 & 65 & 40,4 & 96 & 59,6 & 0,38 & $0,23-0,64$ & $<0,001$ \\
\hline & & & & & & $0,41 *$ & $0,24-0,69$ & \\
\hline Falta de informação & 117 & 58 & 49,6 & 59 & 50,4 & 0,55 & $0,32-0,96$ & \\
\hline Com renda & 122 & 78 & 63,9 & 44 & 36,1 & 1,00 & & \\
\hline \multicolumn{9}{|l|}{ Idade da mãe (anos) } \\
\hline$<20$ & 70 & 25 & 35,7 & 45 & 64,3 & 0,49 & $0,28-0,87$ & 0,012 \\
\hline$\geq 20$ & 324 & 172 & 53,1 & 152 & 46,9 & 1,00 & & \\
\hline
\end{tabular}

OR = Odds ratio; IC95\% = Intervalo de confiança de $95 \%$ do odds ratio; $\mathrm{p}=$ significância (teste qui-quadrado); * Odds ratio ajustado;

Fonte: Sistema de Informações sobre Nascidos Vivos. Centro de Saúde São Quirino: janeiro a dezembro, 1999. Campinas, SP.

\section{Discussão}

O SINASC tem entre seus objetivos que "... a primeira e mais imediata utilização dos dados produzidos deveria se fazer a nível local, visando não só o conhecimento dos dados, como a já citada vigilância dos recém-nascidos de alto risco" (Melo Jorge et al., 1992: 22). ${ }^{1}$

As DNVs enviadas ao CS estudado em 1999 foram preenchidas em mais de $95 \%$ dos dados solicitados, com exceção das variáveis, grupos de ocupação e situação conjugal, que foram incorporadas às declarações posteriormente. Com isso, pôde-se verificar que a partir delas há a possibilidade de conhecer o perfil das mães e $\mathrm{RN}$ da área de abrangência desse CS e propor ações de saúde para esse grupo. A falta de informação da ocupação da mãe foi maior para as mulheres menores de 20 anos, sendo importante que seja verificado no local porque nesse grupo, tais dados não têm sido informados.
Sendo possível, essas informações devem ser resgatadas e a Secretaria de Saúde deve ser comunicada para que as falhas na coleta das informações sejam corrigidas junto aos hospitais.

De acordo com as DNVs todos os partos da região foram realizadas em ambiente hospitalar sendo aproximadamente $65 \%$ foram realizados pelo SUS.

As mulheres do local, em sua maioria, tinham até dois filhos. De acordo com o Instituto Brasileiro de Geografia e Estatística (IBGE) no censo 2000, a taxa de fecundidade no Brasil e no Sudeste foi de 2,35 e 2,1 filhos respectivamente. ${ }^{7}$ Isto indica que a região apresenta dados compatíveis com o país, que apresenta queda dessa taxa em quase todas as faixas etárias.

Com relação ao tipo de parto, foi verificado que $50,3 \%$ das mulheres foram submetidas a partos operatórios, proporção semelhante à cidade, região e estado de São Paulo $(51,9 \%, 49,8 \%$ e 47,5\%, respectivamente), ${ }^{8}$ mas acima do índice brasileiro $(38,1 \%) .9$ 
Tais índices ultrapassando muito o limite considerado adequado pela Organização Mundial de Saúde $(15 \%)$ e estudos mostram que as indicações de partos cesáreos, na maioria das vezes, não levam em conta critérios técnicos estabelecidos pelos órgãos representativos da ginecologia e obstetrícia, mas sim, uma preferência do cliente e do obstetra e a conveniência da cirurgia para se proceder a uma laqueadura tubária. 10 Isso, aumenta o risco de morbimortalidade materna e do RN, além de acarretar aumento de custos. ${ }^{11}$ Neste estudo evidenciaram-se dois grupos protegidos quanto ao parto operatório: as mulheres sem renda as que completaram o primeiro grau. Pode-se supor que isto seja decorrente da realização desses partos em hospitais públicos, onde acontece um número maior de partos naturais. No entanto, serão necessários estudos posteriores para que sejam melhor compreendidas as razões para a ocorrência desse fato.

Os resultados referentes à duração da gestação mostraram que a $6,5 \%$ das crianças nascem com menos de 37 semanas, sendo os dados de prematuridade iguais aos verificados na cidade do Rio de Janeiro, RJ, em 1996,12 menores que os do Brasil em 1998 (7,8\%), 10 mas acima dos de Campinas em 1999 $(5,4 \%)^{13}$ e de Blumenau, SC, em 1997 (4,9\%).14 Embora não haja muita diferença de outras localidades brasileiras, é importante ressaltar que a prematuridade pode contribuir para a mortalidade perinatal e que as situações que desencadeiam este problema podem ser reconhecidas durante a gestação, o que reforça a importância do investimento do serviço de saúde para garantir o acesso ao pré-natal, estimular sua procura pelas gestantes e assegurar sua qualidade.

Quanto ao peso de nascimento, este estudo mostrou uma porcentagem de baixo peso ao nascer de $9,0 \%$, dados estes semelhantes aos do município $(8,3 \%)$ e estado (8,5\%). 15 Assim como a prematuridade, o peso de nascimento exerce influência sobre as chances de sobrevivência na infância, bem como a possibilidade da criança ter um crescimento e desenvolvimento sadios. 16 A proporção de baixo peso do local estudado, embora semelhante aos da região, está bastante acima de países desenvolvidos que giram em torno de 4 a $5 \% 17$ sendo meio mais eficaz para reduzir tal índice a adequada atenção durante a gestação.

Quanto à freqüência ao pré-natal verificou-se que 44,3\% das mulheres não cumpriram o calendário proposto pelos órgãos oficiais, proporção semelhante à do município $(39,4 \%)$ e pouco abaixo de outras regiões do estado de São Paulo (51,2\%). ${ }^{18}$ De acordo com o Programa de Assistência Integral à Saúde da Mulher, para que a gravidez transcorra com segurança são necessários cuidados com a gestante e a atenção básica deve incluir a prevenção, a promoção à saúde o e tratamento de problemas que ocorrem durante o período gestacional e após o parto. Para isso, a mulher deve iniciar o pré-natal ainda no primeiro trimestre de gravidez, fazendo consultas médicas ou de enfermagem mensalmente até 28 semanas, quinzenalmente até 36 semanas e semanalmente até o final da gestação. ${ }^{19}$ Ou seja, para um adequado acompanhamento pré-natal a mulher deve fazer mais do que seis consultas durante a gestação. No entanto, seja por desinformação das mulheres ou por falha da equipe de saúde, nesse CS essa meta não foi cumprida em 1999. Embora neste estudo não tenha sido encontrada associação entre baixo peso de nascimento, prematuridade e freqüência ao prénatal, acredita-se que o acesso ao serviço de saúde deva ser facilitado para que se possa detectar problemas que podem ser resolvidos durante a gravidez.

Com relação às variáveis sócio-demográficas, verificou-se que $26,0 \%$ das mulheres não têm companheiros fixos e que somente $30,5 \%$ delas exercem atividade remunerada. No Brasil as mulheres têm contribuído cada vez mais para a formação do orçamento familiar, quando não assumem a própria economia da família, 10 mas esta situação de pequena inserção no mercado de trabalho e da impossibilidade de dividir o sustento familiar com um companheiro pode comprometer a qualidade de vida da mãe, da criança e dos outros familiares.

Quanto à escolaridade, verificou-se que mais da metade das mulheres $(54,9 \%)$ não concluíram o ensino fundamental, taxa semelhante à do Brasil (59,9\%), segundo dados do Censo 2000.7 Essa constatação é preocupante, pois a baixa escolaridade materna tem sido relacionada à morbimortalidade infantil. Estudos têm mostrado relação entre desvantagens nutricionais, e dificuldade de seguir o calendário vacinal, por exemplo, para os filhos de mães com pouca instrução. ${ }^{20-24}$

As variáveis estudadas para todas as mulheres da região mostraram uma situação inquietante, mas quando são referentes às menores de 20 anos, o quadro se torna mais grave. No Brasil a taxa de fecundidade entre as adolescentes vem aumentando, contrariando a tendência observada nas demais idades. ${ }^{25}$ A preocupação com este grupo deve ser grande, pois a adolescência é caracterizada por um complexo processo de desenvolvimento biológico, psicológico e social e a maternidade precoce poderá acarretar alterações psicossociais negativas para a jovem e sua prole.

A porcentagem de mães adolescentes foi de 
$17,8 \%$, semelhante a de Campinas $(18,8 \%)$ e abaixo da região $(20,1 \%)$, estado $(19,8 \%)$ e país $(23,4 \%) .26$

Essas adolescentes têm menos companheiros fixos do que as adultas, somente $15,4 \%$ delas estão inseridas no mercado de trabalho, $21,4 \%$ têm mais de um filho e $75,6 \%$ têm primeiro grau completo. Os dados da regressão logística indicam que existe um maior risco de ser mãe antes de 20 anos para as mulheres sem companheiro, sem renda e para aquelas onde não há informação sobre a ocupação. Tais achados evidenciam uma situação de risco que pode comprometer a saúde da adolescente e de seus dependentes.

Essa preocupação é reforçada em estudo realizado no Rio de Janeiro, no qual verificou-se que a experiência da gravidez na adolescência agrava as condições desfavoráveis de vida, levando a um aumento no número de gravidezes, maior número de abortamentos, consumo de cigarros e drogas ilícitas na gestação. 27 Além disso, segundo a Academia Americana de Pediatria, os filhos de mães adolescentes têm maior risco de se tornarem pais na adolescência, apresentarem atraso de desenvolvimento, dificuldades escolares, perturbações comportamentais e tóxico-dependência. 28

\section{Conclusões}

Através deste estudo, pôde-se verificar que as DNV do SINASC são fontes de dados bastante ricas, que permitem conhecer o perfil das mulheres que têm filhos na área de abrangência de um CS, permitindo à equipe o monitoramento da realidade local e o planejamento de intervenções de acordo com as necessidades da população adscrita. Para isso deve-se incentivar e supervisionar o preenchimento correto de todos os itens da declaração

A Unidade de Saúde deve investir na melhoria do pré-natal, garantindo acesso das mulheres ao seguimento adequado. Assim, além de assistir à mulher durante a gestação e o puerpério, diminuindo os riscos para as mães e os bebês, também investe na continuidade do acompanhamento no Programa de Assistência Integral à Saúde da Mulher, principalmente para planejamento familiar e prevenção de câncer de colo e útero.

Quanto às mães adolescentes, sugere-se uma vigilância especial no seguimento de saúde, através de atendimentos na unidade, de parcerias com escolas, entidades não-governamentais e grupos da comunidade, de tal forma que, através de educação em saúde, apoio às necessidades próprias da idade e profissionalização, seja possível contribuir para um futuro melhor desse grupo, podendo ser a gravidez uma decisão posterior e consciente.

\section{Referências}

1. Mello-Jorge MHP, Gotlieb SL, Soboll ML, Baldijão MFA, Latorre MR. O Sistema de Informações sobre Nascidos Vivos, Sinasc. São Paulo: Centro Brasileiro de Classificação de Doenças; 1992.

2. Ministério da Saúde. Estatuto da criança e do adolescente: Projeto Minha Gente. Brasília (DF): O Ministério; 1991.

3. Campinas. Secretaria Saúde. Diretrizes básicas saúde da mulher: Projeto Mulher Viva: educando para uma vida saudável. Campinas: Coordenação do Programa de Saúde da Mulher; 1999.

4. Campinas. Secretaria de Saúde. Manual da criança. Campinas: A Secretaria; 1996.

5. Antonio MAGM, Franco SC, Zanolli ML, Carniel EF. Vigilância à saúde para alunos de $4^{\circ}$ ano de Medicina. Rev Bras Educ Med 1999; 23: 90-4.

6. Franco SC, Carniel EF, Antônio MAGM, Zanolli ML. Relato de uma experiência de ensino em vigilância à saúde para residentes de pediatria. Rev Bras Educ Med 1999; 23: 46-52.

7. Góis A. Censo 2000. Folha São Paulo 2002 maio 5; Sect A, Cad Especial: 5.

8. Campinas. Secretaria de Saúde. Número de nascidos vivos por ano e tipo de parto: mães residentes em Campinas 1994 a 2001. Disponível em: http://www.campinas.sp. gov.br/saude/dados/nasc vivos ano tipo parto 94 01.htm. [2002 fev 5].

9. Ministério da Saúde. Nascidos vivos: São Paulo, 2002. Disponível em: http://tabnet. datasus.gov.br/cgi/tabcgi. exe/sinasc/cnv/nvsp.def.; http://www.campinas.sp.gov. br/saude/dados/nasc vivos ano tipo parto 94 01.htm. [2002 fev 5].

10. Correia LL, McAuliffe J. Saúde materno-infantil. In: Rouquayrol MZ, Almeida Filho N. Epidemiologia e saúde. Rio de Janeiro: Medsi; 1999. p. 375-401.

11. Pires HMB, Cecatti JG, Faúndes JG. Fatores associados à prova de trabalho de parto em primíparas com cesárea anterior. Rev Saúde Pública 1999; 33: 342-8.

12. Silva RI, Filha MMT, Noronha CP. Sistema de informações de nascidos vivos na cidade do Rio de Janeiro 1993/1996. Inf Epidemiol SUS 1997; 6: 33-48.

13. Campinas. Secretaria de Saúde. Número de nascidos vivos por ano e duração da gestação: mães residentes em Campinas 1994 a 2001. Disponível em: http://www.campinas. sp.gov.br /saude/da dos/nasc 
vivos ano duracao da gestacao 94 01.htm. [2002 fev 5].

14. Santa Helena, ET, Wisbeck J. Implantação do Sinasc e perfil dos nascidos vivos de Blumenau, 1994-1997. Inf Epidemiol SUS 1998; 7: 35-42.

15. Campinas. Secretaria de Saúde. Número de nascidos vivos por ano e peso ao nascer : mães residentes em Campinas 1994 a 2001. Disponível em: http://www.campinas sp.gov.br/saude/dados/nasc vivos ano peso ao nascer 94 01.htm. [2002 fev 5].

16. Bicalho-Moriotoni GG, Barros Filho AA. Nascer em Campinas: análise de dados do SINASC. Rev Paul Pediatr 1997; 15: 24-30.

17. UNICEF (Fundo das Nações Unidas para a Infância). Relatório da situação da infância: 1998. Brasília (DF): O Fundo; 1998.

18. Campinas. Secretaria de Saúde. Número de nascidos vivos por ano por número de consultas de pré-natal: mães residentes em Campinas 1994 a 2001. Disponível em: http://www.campinas.sp.gov.br/saude/dados/nasc vivos _pre-natal94_01.htm. [2001 fev 5].

19. Ministério da Saúde. Assistência pré-natal: manual técnico. Brasília (DF): O Ministério; 2000.

20. Monteiro CA, Conde WL. Tendência secular da desnutrição e obesidade na infância na cidade de São Paulo: 19741996. Rev Saúde Pública 2000; 34 Supl 6: 52-61.

21. BEMFAM (Sociedade Civil Bem-Estar Familiar no Brasil). Pesquisa Nacional sobre Demografia e Saúde. Brasília (DF): A Sociedade; 1997.
22. Ribas DLB, Philippi ST, Tanaka A, Zorzatto JR. Saúde e estado nutricional de uma população do Centro-Oeste do Brasil. Rev Saúde Pública 1999; 33: 358-65.

23. Antonio MARG, Morcillo AM, Piedrabuena E, Carniel EF. Análise do perfil de crescimento de 566 crianças com idade entre 3 meses e 3 anos matriculados nas 14 creches municipais de Paulínia. J Pediatr [Rio de Janeiro] 1996; 72: $245-50$

24. Silva AAM, Gomes UA, Tonial, SR, Silva RA. Cobertura vacinal e fatores de risco associados à não-vacinação em localidade urbana no Nordeste brasileiro: 1994. Rev Saúde Pública 1999; 33: 147-56.

25. Ministério da Saúde. Assistência à saúde da mulher do ciclo gravídico-puerperal: SUS 1994-1997. Disponível em: http://www.saude.gov.br/Programas/mulher/assist. htm\#pnatal. [2002 fev 5].

26. Campinas. Secretaria de Saúde. Número de nascidos vivos por ano e idade materna: mães residentes em Campinas 1994 a 2001. Disponível em URL: http://www.campinas.sp.gov.br/saude/dados/nasc vivos/nasc vivos idade_materna_94_01.htm. [2002 fev 5].

27. Gama SGN, Szwarcwald CL, Leal MC. Experiência de gravidez na adolescência, fatores associados e resultados peri-natais em puérperas de baixa renda. Cad Saúde Pública 2002; 18: 153-61.

28. Academia Americana de Pediatria. Gravidez na adolescência: tendências e questões atuais. Pediatrics [ed. bras] 1999; 3: 439-44.

Recebido em 28 de janeiro de 2002

Versão final reapresentada em 28 abril de 2003

Aprovado em 10 de maio de 2003 